\section{A CALL FOR HELP}

\section{H.N. Hart, M.D., Ph.D., Rotterdam, The Netherlands}

In 1985, a film was presented on Dutch television on the occasion of a project which offered assistance by telephone of the central dispatch nurse. This can be accessed when a potential assisting bystander fears a cardiac arrest in the home.

The film shows how a dispatch nurse can provide protocol information on how to perform CPR while waiting for the ambulance to arrive.

In Seattle this system has been used more than four years and has saved many lives.

The second part of the film shows how in Rotterdam, Holland, this project began by testing a preliminary telephone protocol on volunteers.

\section{THE ROTTERDAM CARDIOPULMONARY RESUSCITATION TRAINING PROGRAM FOR LAY PERSONS: RESULTS OF AN EVALUATION}

\section{H.N. Hart, M.D., Ph.D. and R. Sloof, Ph.D., Rotterdam, The Netherlands}

A continuing training program in cardiopulmonary resuscitation (CPR) for lay persons was established by the Foundation EHBH in June, 1979. Since then, about 32,000 Rotterdam citizens have been trained in the technique. One year after each course, an evaluation questionnaire was mailed to all 7,880 persons trained between June 1979 and July 1981 . Among the 5,312 respondents $(67 \%), 109$ had practiced CPR in the preceding year. Of these, reasonably complete personal data were available for 105 , of whom 99 were questioned verbally on the circumstances of the event, on the nature of the incident and on the identity of the patient. Using the case-control type approach, a systematic sample of 221 trainees from the same period was used as a control group to investigate which personal characteristics were associated with the actual practice of CPR. This kind of information was considered important for formulating a target group policy for the program.
The results showed that profession was the most important explanatory variable. Trainees in educational or recreational and governmental jobs resuscitated 7.6 times as often as professionals in the group resuscitating the least (administrative or financial). Males resuscitated 3.5 times as often as females. Trainees with a First Aid certificate resuscitated 6.5 times as often as trainees without such a certificate and trainees below 32 years of age twice as often as trainees older than 31 years.

\section{AUSTRALIAN RESUSCITATION COUNCIL}

\section{Harry F. Oxer, M.D., Belmont, Australia}

The Australian Resuscitation Council was set up and sponsored ten years ago by the Royal Australian College of Surgeons. Its aim is to foster and coordinate the teaching and practice of resuscitation, and to promote uniformity and standardization.

During the early years, most work was aimed towards achieving consensus, and publishing recommendations on resuscitation. The work was hampered by lack of funds.

Recently a Federal Grant was received and review, update and reprinting of policies is being carried out. Promotion of resuscitation training is being actively pursued. The Australian Resuscitation Council is now widely accepted as the authoritative reference body in its field of interest.

\section{THE STRETCHER: YESTERDAY AND TODAY}

\section{Svein Dommerud, M.D., Nesbru, Norway}

Since humans started hurting each other, three ways of treating the victims have been used: comforting, dressing and carrying on stretchers. The medical treatment has undergone great changes, but the stretcher has remained the same. This in spite of the fact that the stretcher is one of the most important tools in emergency and disaster medicine. It is indispensable, even for untrained persons.

Today, a patient spends comparatively little time being carried by hand on a stretcher. Most of the time he is waiting for or receiving treatment.

Minimum requirements for a modern stretcher are that it shall be long enough, light and strong. The 